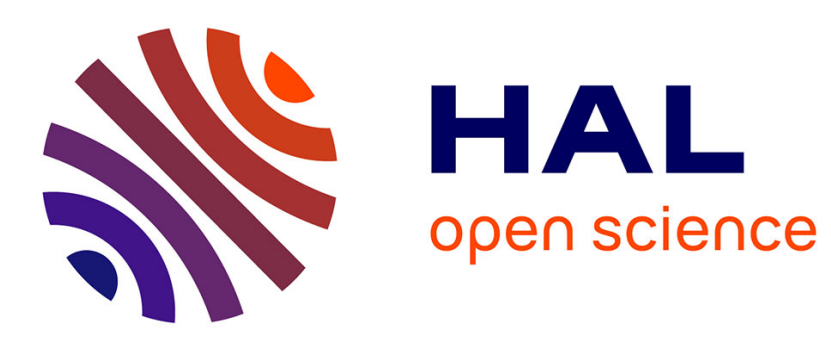

\title{
Refined IV-based method for LPV partial differential equation model identification
}

\author{
Julien Schorsch, Vincent Laurain, Marion Gilson, Hugues Garnier
}

\section{To cite this version:}

Julien Schorsch, Vincent Laurain, Marion Gilson, Hugues Garnier. Refined IV-based method for LPV partial differential equation model identification. 13th European Control Conference, ECC'14, Jun 2014, Strasbourg, France. pp.2127-2132. hal-01058618

\section{HAL Id: hal-01058618 https://hal.science/hal-01058618}

Submitted on 27 Aug 2014

HAL is a multi-disciplinary open access archive for the deposit and dissemination of scientific research documents, whether they are published or not. The documents may come from teaching and research institutions in France or abroad, or from public or private research centers.
L'archive ouverte pluridisciplinaire HAL, est destinée au dépôt et à la diffusion de documents scientifiques de niveau recherche, publiés ou non, émanant des établissements d'enseignement et de recherche français ou étrangers, des laboratoires publics ou privés. 


\title{
Refined IV-based method for LPV partial differential equation model identification
}

\author{
Julien Schorsch ${ }^{1,2}$, Vincent Laurain ${ }^{1,2}$, Marion Gilson ${ }^{1,2}$ and Hugues Garnier ${ }^{1,2}$
}

\begin{abstract}
This paper presents a direct identification method for linear parameter varying models described by partial differential equations in an input-output setting. The continuous space-time model is firstly rewritten as a multiple-input singleoutput model. The continuous filtering operations are reformulated as a discrete convolution product and a refined instrumental variable technique is developed to efficiently estimate the model parameters. The performance of the proposed method is then illustrated via a representative simulation example.
\end{abstract}

\section{INTRODUCTION}

The linear parameter varying (LPV) partial differential equation (PDE) identification case is considered in this paper where the parameters are assumed to be function of a measurable deterministic time-space varying signal. This modeling concept allows for a wide representation of physical processes.

Several identification methods for PDE models are available in the literature (see e.g. [6] for survey) and two main approaches can be distinguished. The indirect approach including two steps involves the transformation of the continuous time-space (CTS) model into a discrete timespace (DTS) model and the identification of the DTS model parameters [1]. The direct approach aims to estimate the coefficients of the CTS model directly from sampled signals ([4] and [12]). However, only a few papers deal with the PDE identification problem into the LPV framework. The use of LPV models has been applied to the identification of linear time invariant (LTI) PDE system by approximating the PDE at each working point with a DT-LPV model [2].

A method has been already proposed to identify a continuous space-time LPV partial differential equation model [13]. The approach is based on an iterative procedure develop to estimate efficiently the model parameters into the errorprediction minimization framework. However, as it will be shown in the numerical example developed in this paper, in the situation where the output is corrupted by an additive colored noise, this method delivers biased estimates.

The proposed direct approach uses here an instrumental variable (IV) based method which provides consistent parameter estimates whatever the kind of noise [14] or [16]. The Simplified Refined Instrumental Variable for Continuoustime systems (SRIVC) method was recently extended to continuous-time LPV ordinary differential equation (ODE)

\footnotetext{
${ }^{1}$ The authors are with University of Lorraine, CRAN, UMR 7039, 2 rue Jean Lamour, F-54519 Vandœuvre-les-Nancy, France; ${ }^{2}$ CNRS, CRAN, UMR 7039, France

Email adresses: julien.schorsch@univ-lorraine.fr; vincent.laurain@univlorraine.fr; marion.gilson@univ-lorraine.fr; hugues.garnier@univ-lorraine.fr
}

models [7] and to linear PDE models [12]. It is here adapted for the LPV-PDE models.

An advantage of the SRIVC algorithm is to make use of a filter which reduces the noise influence on the estimated parameters. In the ODE model case, this filter follows the same dynamic as the system. However, in the LPV-PDE case, the filter should be dependent on an external variable $\rho$, if it is chosen to follow the same dynamics as the system. The influence of this dependency is twofold. First, this dependency is injected on the filtered noise which decreases the quality of the estimated parameters. Secondly, the classical algebraic properties (commutativity, associativity,...) are not guaranteed. The proposed solution is based on the reformulation of the single-input single-output (SISO) LPVPDE model to a multiple-input single-output (MISO) PDE model. From this reformulation a new filter is emphasized. It is not anymore dependent on $\rho$, keeps algebraic properties and follows a dynamic part of the considered system.

However, the resulting filter is written in terms of partial derivative operators and therefore it cannot be directly defined as a rational transfer function. Usually, the transfer functions associated with PDE models are irrational functions which involve an infinite number of poles and zeros [5]. Nevertheless, the solution developed here, is to discretize the filter by using classical numerical schemes and to write the filter operations as a convolution product.

The paper is organized as follows: in Section 2, the general class of CTS-LPV-PDE models is introduced. The reformulation of the system is provided Section 3 including a description of the proposed filter. Section 4 deals with the writing of this filter by using the discretization of the differential operator. A description of the proposed estimator is given in Section 5. Finally, an numerical example is used in Section 6 to illustrate the performance of the proposed approach.

\section{Problem fORMUlation}

\section{A. Deterministic model}

Let $\Omega$ a close set and consider the continuous model described by the following PDE:

$$
\left\{\begin{array}{l}
A(\partial, \rho) \dot{\chi}(t, x)=B(\partial, \rho) u(t, x) \text { on } \Omega \times] 0,+\infty[ \\
\dot{\chi}(0, x)=\phi(x) \text { on } \Omega
\end{array}\right.
$$

$x \in \Omega$ is the space variable, note that for clarity of the presentation, $x$ is supposed to be one-dimensional. $t \in[0, T]$ is the time variable. $\dot{\chi}(x, t)$ is the model output and $u(x, t)$ is the input. $\phi(x)$ is a continuous space function which 
represents the model behaviour for $t=0$. For $\left(i_{t}, i_{x}\right) \in \mathbb{N}^{2}$, $\partial^{i_{t}, i_{x}}$ denotes the partial differential operator:

$$
\partial^{i_{t}, i_{x}} \stackrel{\circ}{\chi}(t, x)=\partial_{t}^{i_{t}} \partial_{x}^{i_{x}} \stackrel{\circ}{\chi}(t, x)=\frac{\partial^{i_{t}+i_{x}} \stackrel{\circ}{\chi}(t, x)}{\partial t^{i_{t}} \partial x^{i_{x}}},
$$

where $\partial_{t}^{i_{t}}=\frac{\partial^{i} t}{\partial t^{i} t}$ is the $i_{t}$-th time partial derivative and $\partial_{x}^{i_{x}}=\frac{\partial^{i_{x}}}{\partial x^{i_{x}}}$ is the $i_{x}$-th space partial derivative.

To complete Equation (1) boundary conditions needs to be specify. A large number of boundary conditions can be chosen, depending on the problem formulation, the number of variables involved and the nature of the equation.

$A(\partial, \rho)$ and $B(\partial, \rho)$ are bivariate polynomials in $\partial$ of degrees $n_{t}, n_{x}$ and $m_{t}, m_{x}$ respectively:

$$
\left\{\begin{array}{l}
A(\partial, \rho)=p^{n_{t}, n_{x}}+\sum_{i_{t}, i_{x}=0}^{\substack{\left.n_{t}, n_{x} \\
i_{t}, i_{x}\right) \neq\left(n_{t}, n_{x}\right)}} a(\rho)_{i_{t}, i_{x}} \partial^{i_{t}, i_{x}} \\
B(\partial, \rho)=\sum_{j_{t}, j_{x}=0}^{m_{t}, m_{x}} b(\rho)_{j_{t}, j_{x}} \partial^{j_{t}, j_{x}}
\end{array}\right.
$$

In order to represent a larger behavior range, the model is assumed to be parameter varying. $a_{i_{t}, i_{x}}(\rho)$ and $b_{j_{t}, j_{x}}(\rho)$ are analytic functions with dependence on an external deterministic scheduling variable $\rho(t, x)=\rho$, which is called static dependence. $\left(n_{t}, n_{x}\right)$ are the orders of the system (assumed to be known). In this work, it is assumed that $n_{x} \geq m_{x}, m_{t}$; $n_{t} \geq m_{x}, m_{t}$; and $A(\partial, \rho)$ is a monic polynomial in $\partial_{t}$ variable.

\section{B. Process model}

Assuming $\rho$ a priori known, the $A(\partial, \rho)$ and $B(\partial, \rho)$ polynomials can be parametrized as:

$$
\begin{aligned}
& \left\{\begin{array}{c}
A(\partial, \rho, \theta)=\partial^{n_{t}, n_{x}}+\sum_{i_{t}, i_{x}=0}^{\substack{n_{t}, n_{x} \\
\left(i_{t}, i_{x}\right) \neq=\left(n_{t}, n_{x}\right)}} a_{i_{t}, i_{x}}(\rho) \partial^{i_{t}, i_{x}} \\
a_{i_{t}, i_{x}}(\rho)=a_{i_{t}, i_{x}}^{0}+\sum_{l=1}^{n_{\alpha}} a_{i_{t}, i_{x}}^{l} \mathrm{f}_{l}(\rho) \\
i_{t}=0, \cdots, n_{t} i_{x}=0, \cdots, n_{x}
\end{array}\right. \\
& \left\{\begin{aligned}
B(\partial, \rho, \theta) & =\sum_{j_{t}, j_{x}=0}^{m_{t}, m_{x}} b_{j_{t}, j_{x}}(\rho) \partial^{j_{t}, j_{x}} \\
b_{j_{t}, j_{x}}(\rho)= & b_{j_{t}, j_{x}}^{0}+\sum_{l=1}^{m_{\beta}} b_{j_{t}, j_{x}}^{l} \mathrm{~g}_{l}(\rho) \\
& j_{t}=0, \cdots, m_{t} \quad j_{x}=0, \cdots, m_{x} .
\end{aligned}\right.
\end{aligned}
$$

In this parametrization, $\left\{\mathrm{f}_{l}\right\}_{l=1}^{n_{\alpha}}$ and $\left\{\mathrm{g}_{l}\right\}_{l=1}^{m_{\beta}}$ are meromorphic functions of $\rho$, with static dependence. They can be chosen for example as linearly independent functions. The associated model parameters $\theta$ are stacked columnwise:

$\theta=\left[\begin{array}{llllll}a_{0,0} & \cdots & a_{n_{t}, n_{x}-1} & b_{0,0} & \cdots & b_{m_{t}, m_{x}}\end{array}\right]^{\mathrm{T}} \in \mathbb{R}^{n_{\theta}}$

where

$$
a_{i_{t}, i_{x}}=\left[\begin{array}{llll}
a_{i_{t}, i_{x}}^{0} & a_{i_{t}, i_{x}}^{1} & \cdots & a_{i_{t}, i_{x}}^{n_{\alpha}}
\end{array}\right] \in \mathbb{R}^{n_{\alpha}+1}
$$

$$
b_{j_{t}, j_{x}}=\left[\begin{array}{llll}
b_{j_{t}, j_{x}}^{0} & b_{j_{t}, j_{x}}^{1} & \cdots & b_{j_{t}, j_{x}}^{m_{\beta}}
\end{array}\right] \in \mathbb{R}^{m_{\beta}+1}
$$

and $n_{\theta}=\left(\left(n_{t}+1\right)\left(n_{x}+1\right)-1\right)\left(n_{\alpha}+1\right)+\left(m_{t}+1\right)\left(m_{x}+\right.$ 1) $\left(m_{\beta}+1\right)$.

\section{Noisy model}

In this paper, stochastic PDE class is considered. These systems are a generalization of PDE taking into account a random noise term. Thus due to measurement inaccuracies, the measure $\dot{\chi}(t, x)$ is not available but a disturbed version of it is obtained.

Moreover, in practical situations, such as those encountered in environmental science, the measured data are not continuous in time and in space. Given the sampled nature of the data, an obvious assumption is that the sampled output is contaminated by a discrete time-space noise.

The data-generating system takes the following OutputError structure:

$$
\left\{\begin{array}{l}
A(\partial, \rho, \theta) \stackrel{\circ}{\chi}(t, x)=B(\partial, \rho, \theta) u(t, x) \text { on } \Omega \times] 0, T] \\
\grave{\chi}(0, x)=\phi(x) \text { on } \Omega \\
y\left(t_{k}, x_{\ell}\right)=\stackrel{\circ}{\chi}\left(t_{k}, x_{\ell}\right)+e\left(t_{k}, x_{\ell}\right)
\end{array}\right.
$$

where the observed noisy output $y\left(t_{k}, x_{\ell}\right)$ and $\dot{\chi}\left(t_{k}, x_{\ell}\right)$ are the sampled signals at the time $t_{k}$ and the observation point $x_{\ell}$ for $k \in\{1, \cdots, N\}$ and $\ell \in\{1, \cdots, L\} . e\left(t_{k}, x_{\ell}\right)$ is a white noise, i.e. a zero mean, random spatial array, with no temporal or spatial correlation.

\section{Objective}

Finally, the objective is to estimate the parameter vector (6) of the model (9) based on the measured sampled input, output data and the scheduling variables $\mathcal{Z}=$ $\left\{u\left(t_{k}, x_{\ell}\right), y\left(t_{k}, x_{\ell}\right), \rho\left(t_{k}, x_{\ell}\right)\right\}_{\ell=1, k=1}^{L, N}$.

\section{THE LPV-PDE REFORMULATION}

\section{A. Reformulation}

The LPV-PDE model identification problem cannot be formulated directly as a prediction error minimisation because of the non-commutativity of the LPV polynomials. This problem is well-known when dealing with LPV models. In [7] (for ODE models) and [13] (for PDE models), the proposed solution is to rewrite the LPV model as a MISO LTI model for which the PEM can be formulated. The idea here is to follow the same concept and to reformulate the LPV model in order to express it as a linear regression form:

$$
y^{\left(n_{t}, n_{x}\right)}\left(t_{k}, x_{\ell}\right)=\varphi\left(t_{k}, x_{\ell}\right) \theta+F(\partial, \theta) e\left(t_{k}, x_{\ell}\right)
$$


Such a reformulation can be expressed as:

$$
\begin{aligned}
& \underbrace{\dot{\chi}^{\left(n_{t}, n_{x}\right)}\left(t_{k}, x_{\ell}\right)+\sum_{i_{t}, i_{x}=0}^{\substack{n_{t}, n_{x} \\
\left(i_{t}, i_{x}\right) \neq\left(n_{t}, n_{x}\right)}} a_{i_{t}, i_{x}}^{0} \dot{\chi}^{\left(i_{t}, i_{x}\right)}\left(t_{k}, x_{\ell}\right)}_{F(\partial, \theta) \dot{\chi}\left(t_{k}, x_{\ell}\right)} \\
& +\sum_{i_{t}, i_{x}=0}^{\substack{\left.i_{t}, i_{x}\right) \neq=\left(n_{t}, n_{x}\right) \\
i_{t}, n_{x}}} \sum_{l=1}^{n_{\alpha}} a_{i_{t}, i_{x}}^{l} \underbrace{\mathrm{f}_{l}(\rho) \dot{\chi}^{\left(i_{t}, i_{x}\right)}\left(t_{k}, x_{\ell}\right)}_{\dot{\chi}_{l}^{\left(i_{t}, i_{x}\right)}\left(t_{k}, x_{\ell}\right)} \\
& =\sum_{j_{t}, j_{x}=0}^{m_{t}, m_{x}} \sum_{l=0}^{m_{\beta}} b_{j_{t}, j_{x}}^{l} \underbrace{g_{l}(\rho) u^{\left(j_{t}, j_{x}\right)}\left(t_{k}, x_{\ell}\right)}_{u_{l}^{\left(j_{t}, j_{x}\right)}\left(t_{k}, x_{\ell}\right)}
\end{aligned}
$$

where $\mathrm{g}_{0}(\rho)=1$ and the superscript $\left(j_{t}, j_{x}\right)$ for a signal, like $u^{\left(j_{t}, j_{x}\right)}$, denotes the $j_{t}^{\text {th }}$ partial derivative in time and the $j_{x}^{\text {th }}$ partial derivative in space and

$$
F(\partial, \theta)=\partial^{n_{t}, n_{x}}+\sum_{i_{t}, i_{x}=0}^{\substack{n_{t}, n_{x} \\\left(i_{t}, i_{x}\right) \neq\left(n_{t}, n_{x}\right)}} a_{i_{t}, i_{x}}^{0} \partial^{i_{t}, i_{x}}
$$

Note that in this way, the main advantage is to transpose the time-space variation of the coefficients onto the signals now expressed as

$$
\left\{\begin{array}{c}
\dot{\chi}_{l}^{\left(i_{t}, i_{x}\right)}\left(t_{k}, x_{\ell}\right)=\mathrm{f}_{l}(\rho) \dot{\chi}^{\left(i_{t}, i_{x}\right)}\left(t_{k}, x_{\ell}\right) \\
\left\{i_{t}, i_{x}, l\right\} \in\left\{0 \cdots n_{t}, 0 \cdots n_{x}, 1 \cdots n_{\alpha}\right\} \\
u_{l}^{\left(j_{t}, j_{x}\right)}\left(t_{k}, x_{\ell}\right)=\mathrm{g}_{l}(\rho) u^{\left(j_{t}, j_{x}\right)}\left(t_{k}, x_{\ell}\right) \\
\left\{j_{t}, j_{x}, l\right\} \in\left\{0 \cdots m_{t}, 0 \cdots m_{x}, 0 \cdots m_{\beta}\right\}
\end{array}\right.
$$

Therefore, the process part of the LPV model is rewritten as a MISO system with $\left(\left(n_{t}+1\right)\left(n_{x}+1\right)-1\right) n_{\alpha}+$ $\left(m_{t}+1\right)\left(m_{x}+1\right)\left(m_{\beta}+1\right)$ inputs $\left\{\chi_{i_{t}, i_{x}}^{l}\right\}_{i_{t}=0, i_{x}=0, l=1}^{n_{t}, n_{x}, n_{\alpha}}$ and $\left\{u_{j_{t}, j_{x}}^{l}\right\}_{j_{t}=0, j_{x}=0, l=0}^{m_{t}, m_{x}, m_{\beta}}$. By using (11), (9) can be rewritten in terms of the output signal $y\left(t_{k}, x_{\ell}\right)$ as

$$
\left\{\begin{array}{l}
F(\partial, \theta) y\left(t_{k}, x_{\ell}\right)=-\sum_{i_{t}, i_{x}=0}^{\substack{n_{t}, n_{x} \\
\left(i_{t}, i_{x}\right) \neq\left(n_{t}, n_{x}\right)}} \sum_{l=1}^{n_{\alpha}} a_{i_{t}, i_{x}}^{l} \grave{\chi}_{l}^{\left(i_{t}, i_{x}\right)}\left(t_{k}, x_{\ell}\right) \\
+\sum_{j_{t}, j_{x}=0}^{m_{t}, m_{x}} \sum_{l=0}^{m_{\beta}} b_{j_{t}, j_{x}}^{l} u_{l}^{\left(j_{t}, j_{x}\right)}\left(t_{k}, x_{\ell}\right)+F(\partial, \theta) e\left(t_{k}, x_{\ell}\right) .
\end{array}\right.
$$

which can be written under the regression form of (10) where

$$
\begin{aligned}
\varphi\left(t_{k}, x_{\ell}\right)= & {\left[-y^{(0,0)}\left(t_{k}, x_{\ell}\right) \cdots-y^{\left(n_{t}, n_{x}-1\right)}\left(t_{k}, x_{\ell}\right)\right.} \\
& -\dot{\chi}_{1}^{(0,0)}\left(t_{k}, x_{\ell}\right) \cdots-\dot{\circ}_{\left.n_{\alpha}, n_{x}-1\right)}^{\left(n_{n}, n_{k}\right.}\left(t_{k}, x_{\ell}\right) \\
& \left.u_{0}^{(0,0)}\left(t_{k}, x_{\ell}\right) \cdots u_{\left.m_{\beta}, m_{x}\right)}^{\left(m_{k}\right)}\left(t_{k}, x_{\ell}\right)\right]^{\mathrm{T}} .
\end{aligned}
$$

The regressor is made up with the input $u\left(t_{k}, x_{\ell}\right)$, the noisy output $y\left(t_{k}, x_{\ell}\right)$ and noise-free output $\dot{\chi}\left(t_{k}, x_{\ell}\right)$.

\section{B. Linear regression form}

In the given context, contrary to the LPV formulation where the LPV dependency appears into the signal definitions, the filter $F(\partial, \theta)$ commutes so that (10) can be rewritten as

$$
y_{\mathrm{f}}^{\left(n_{t}, n_{x}\right)}\left(t_{k}, x_{\ell}\right)=\varphi_{\mathrm{f}}^{\mathrm{T}}\left(t_{k}, x_{\ell}\right) \theta+e\left(t_{k}, x_{\ell}\right)
$$

where $y_{\mathrm{f}}\left(t_{k}, x_{\ell}\right), \stackrel{\circ}{\chi}_{l, \mathrm{f}}^{\left(i_{t}, i_{x}\right)}\left(t_{k}, x_{\ell}\right)$ and $u_{l, \mathrm{f}}^{\left(j_{t}, j_{x}\right)}\left(t_{k}, x_{\ell}\right)$ represent the outputs of a filtering operation.

$$
y_{\mathrm{f}}\left(t_{k}, x_{\ell}\right)=\frac{1}{F(\partial, \theta)} y\left(t_{k}, x_{\ell}\right)
$$

and

$$
\begin{aligned}
\varphi_{\mathrm{f}}\left(t_{k}, x_{\ell}\right)= & {\left[-y_{\mathrm{f}}^{(0,0)}\left(t_{k}, x_{\ell}\right) \cdots-y_{\mathrm{f}}^{\left(n_{t}, n_{x}-1\right)}\left(t_{k}, x_{\ell}\right)\right.} \\
- & \dot{\circ}_{1, \mathrm{f}}^{(0,0)}\left(t_{k}, x_{\ell}\right) \cdots-\dot{\circ}_{n_{\alpha}, \mathrm{f}}^{\left(n_{t}, n_{x}-1\right)}\left(t_{k}, x_{\ell}\right) \\
& \left.u_{0, \mathrm{f}}^{(0,0)}\left(t_{k}, x_{\ell}\right) \cdots u_{m_{\beta}, \mathrm{f}}^{\left(m_{t}, m_{x}\right)}\left(t_{k}, x_{\ell}\right)\right]^{\mathrm{T}}
\end{aligned}
$$

Moreover, the proposed filter does not depend on the deterministic scheduling variable $\rho$ but only on the LTI part of the PDE. However, since the inverse of $\partial$ is not defined in the partial derivative context, the digital implementation of the filtering operation is discussed in the next Section.

\section{THE DIFFERENTIAL OPERATOR DISCRETIZATION}

In contrary to ODE model which can be expressed by a transfer function model between the excitation signal and output signal, it is more complicated to write PDE under this form. However, for specific linear PDE systems, several approaches are possible. As an example, a method developed in [5] consists in using the Laplace transforms with respect to the time variable $t$ and then to apply it on the system and the boundary conditions. PDE are then written as irrational transfer functions which can be expanded into infinite dimensional rational transfer functions. A different solution is to use fractional models to describe the PDE system (see e.g. [9]). Another method consists in taking the Fourier transforms with respect to the space variable $x$. This method is used when the space domain is assumed to be infinite and permits to obtain an analytic solution of the PDE.

The method used here, to tackle the irrational function problem, is to consider a discretization along the dimension axis, leading to a so-called parameter distributed system. This approach consists in reducing the infinite dimensional system described by PDE to a finite dimensional model, using the input and output observations to construct an approximation of the solution. The continuous filter $F(\partial, \theta)$ is then transformed into a discrete filter $F(\widetilde{\delta}, \theta)$, where $\partial$ is approximated by a discretized operator $\partial=\left(\partial_{t}, \partial_{x}\right)$ :

$$
\left\{\begin{array}{l}
\partial_{t}=\partial_{t}+\mathcal{O}\left(T_{e}\right) \\
\partial_{x}=\check{\partial}_{x}+\mathcal{O}(h)
\end{array}\right.
$$

where $T_{s}$ and $h$ are the sampling periods in time and space and $\mathcal{O}($.$) is the truncation error.$

This discrete filter can be expressed as a convolution product

$$
[F(\check{\partial}, \theta) \grave{\chi}]\left(t_{k}, x_{\ell}\right)=(f * \stackrel{\circ}{\chi})\left(t_{k}, x_{\ell}\right) \text {. }
$$

Using the simplest partial derivatives approximations made by finite difference method in equation (20), the filter can be realized as a finite-dimensional rational transfer function. 


\section{Example:}

Consider the heat equation

$$
\partial^{1,0} \stackrel{\chi}{\chi}(t, x)-\partial^{0,2} \grave{\chi}(t, x)=u(t, x)
$$

$\partial^{1,0} \chi(t, x)$ can be computed by the following Euler forward difference approximation:

$$
\left[\check{\partial}^{1,0} \stackrel{\circ}{\chi}\right]\left(t_{k}, x_{\ell}\right)=\frac{q_{t}^{+1}-1}{T_{s}} \grave{\chi}\left(t_{k}, x_{\ell}\right)
$$

where $q_{t}$ is the shift operator. In the same way, $\partial^{0,2} \chi(t, x)$ can be approximated by the central difference approximation:

$$
\left[\check{\delta}^{0,2} \chi\right]\left(t_{k}, x_{\ell}\right)=\frac{q_{x}^{+1}-2+q_{x}^{-1}}{h^{2}} \grave{\chi}\left(t_{k}, x_{\ell}\right) .
$$

where $q_{x}$ is the shift operator.

Equation (21) can then be expressed into the following discrete form

$$
\left(\check{\partial}^{1,0}-\check{\partial}^{0,2}\right) \dot{\chi}\left(t_{k}, x_{\ell}\right)=u\left(t_{k}, x_{\ell}\right),
$$

which can be written under the following transfer function:

$$
\left\{\begin{array}{l}
G\left(q_{t}, q_{x}\right)=\frac{T_{s}}{q_{t}^{+1} q_{x}^{0}+\frac{T_{s}}{h^{2}} q_{t}^{0} q_{x}^{+1}+\left(1-\frac{2 T_{s}}{h^{2}}\right) q_{t}^{0} q_{x}^{0}+\frac{T_{s}}{h^{2}} q_{t}^{0} q_{x}^{-1}} \\
\grave{\chi}\left(t_{k}, x_{\ell}\right)=G\left(q_{t}, q_{x}\right) u\left(t_{k}, x_{\ell}\right)
\end{array}\right.
$$

The stability of this transfer function can be proven by the classical methods used for studying the convergence of the numerical scheme [10]. Here the transfer function $G\left(q_{t}, q_{x}\right)$ is stable when

$$
-1+\frac{2 T_{s}}{h^{2}} \leq 0 .
$$

\section{ESTIMATION}

Under the assumption of a priori known $F(ð, \theta)$ and $\stackrel{\circ}{\chi}$, the optimal estimator in the prediction error minimization sense is the least-squares (LS) estimator:

$$
\begin{aligned}
& \hat{\theta}_{\mathrm{LS}}=\left[\sum_{k, \ell=1}^{N, L} \varphi_{\mathrm{f}}\left(t_{k}, x_{\ell}\right) \varphi_{\mathrm{f}}^{\mathrm{T}}\left(t_{k}, x_{\ell}\right)\right]^{-1} . \\
& {\left[\sum_{k, \ell=1}^{N, L} \varphi_{\mathrm{f}}\left(t_{k}, x_{\ell}\right) y_{\mathrm{f}}^{\left(n_{t}, n_{x}\right)}\left(t_{k}, x_{\ell}\right)\right]}
\end{aligned}
$$

In practice, the filters $F(ð, \theta)$ and the signals $\dot{\chi}^{\left(i_{t}, i_{x}\right)}$ are unknown and therefore an iterative procedure is proposed in order to cope with this issue, similarly as it is achieved in the approaches developed for the ODE case in [8] and [13] for the PDE case. However, the LS method delivers biased estimates in the general practical situation where the noise structure is not known. It is then necessary to develop estimators which give unbiased estimates whatever the noise. In this paper, the proposed estimator is based on the IV method which is known to provide efficient estimates.

\section{A. Basic IV estimator}

The main idea behind the IV method is to modify the normal equations (27) so that they yield consistent estimates for arbitrary additive noise. This involves the introduction of an IV vector $\zeta_{\mathrm{f}}\left(x_{\ell}, t_{k}\right)$ whose elements are correlated with the noise-free regression vector $\stackrel{\circ}{\mathrm{f}}_{\mathrm{f}}\left(x_{\ell}, t_{k}\right)$, where

$$
\stackrel{\circ}{\mathrm{f}}_{\mathrm{f}}\left(t_{k}, x_{\ell}\right)=F(\check{\partial}, \theta) \stackrel{\circ}{\varphi}\left(t_{k}, x_{\ell}\right)
$$

with

$$
\begin{aligned}
\check{\varphi}\left(t_{k}, x_{\ell}\right)= & {\left[-\dot{\chi}^{(0,0)}\left(t_{k}, x_{\ell}\right) \cdots-\dot{\chi}^{\left(n_{t}, n_{x}-1\right)}\left(t_{k}, x_{\ell}\right)\right.} \\
& -\dot{\chi}_{1}^{(0,0)}\left(t_{k}, x_{\ell}\right) \cdots-\dot{\circ}_{n_{\alpha}}^{\left(n_{t}, n_{x}-1\right)}\left(t_{k}, x_{\ell}\right) \\
& \left.u_{0}^{(0,0)}\left(t_{k}, x_{\ell}\right) \cdots u_{m_{\beta}}^{\left(m_{t}, m_{x}\right)}\left(t_{k}, x_{\ell}\right)\right]^{\mathrm{T}}
\end{aligned}
$$

but uncorrelated with the additive noise. The basic IV parameter estimates are then given by the following IV normal equations:

$$
\begin{aligned}
& \hat{\theta}_{\mathrm{IV}}=\left[\sum_{k, \ell=1}^{N, L} \zeta_{\mathrm{f}}\left(t_{k}, x_{\ell}\right) \varphi_{\mathrm{f}}^{\mathrm{T}}\left(t_{k}, x_{\ell}\right)\right]^{-1} . \\
& {\left[\sum_{k, \ell=1}^{N, L} \zeta_{\mathrm{f}}\left(t_{k}, x_{\ell}\right) y_{\mathrm{f}}^{\left(n_{t}, n_{x}\right)}\left(t_{k}, x_{\ell}\right)\right]}
\end{aligned}
$$

provided that the matrix-inverse exists.

\section{B. Consistency properties}

By introducing (16) into (30) it comes:

$$
\begin{array}{r}
\hat{\theta}_{\mathrm{IV}}=\theta+\left[\sum_{k, \ell=1}^{N, L} \zeta_{\mathrm{f}}\left(x_{\ell}, t_{k}\right) \varphi_{\mathrm{f}}^{\mathrm{T}}\left(x_{\ell}, t_{k}\right)\right]^{-1} \cdot \\
{\left[\sum_{k, \ell=1}^{N, L} \zeta_{\mathrm{f}}\left(x_{\ell}, t_{k}\right) e_{\mathrm{f}}\left(t_{k}, x_{\ell}\right)\right]}
\end{array}
$$

It can be deduced from (31) that $\hat{\theta}_{\mathrm{IV}}$ is a consistent estimate of $\theta$ if

$$
\left\{\begin{array}{l}
E\left[\zeta_{\mathrm{f}}\left(t_{k}, x_{\ell}\right) \varphi_{\mathrm{f}}^{\mathrm{T}}\left(t_{k}, x_{\ell}\right)\right] \quad \text { is not singular } \\
E\left[\zeta_{\mathrm{f}}\left(t_{k}, x_{\ell}\right) e_{\mathrm{f}}\left(t_{k}, x_{\ell}\right)\right]=0
\end{array}\right.
$$

where $E($.$) denotes the mathematical expectation.$

\section{Refined Instrumental Variable algorithm}

Theoretically, the IV method corrects the statistical inconsistency of the least squares solution but its practical implementation requires some method for generating an instrument that satisfies the conditions given by (32a) and (32b). Several different procedures for the ODE models have been suggested in the system and control literature. The most successful of these is the statistically optimal SRIVC algorithm which involves an iterative procedure where at each iteration, the auxiliary model used to generate the IV, as well as the associated filters are updated based on the parameter estimates at the previous iteration.

The proposed LPV-SRIVC-PDE model identification algorithm can be summarized as shown below. The algorithm consists of three first steps for the initialization and the 
last five iterative steps are used to estimate the filter, the derivatives and finally the model parameters.

- Step 1 Initialization of $\theta^{0}$ for $l=0$ only:

$$
\hat{\theta}^{0}=\left[\hat{a}_{0,0}^{0} \cdots \hat{a}_{n_{t}, n_{x}-1}^{0} 0 \cdots 0 \hat{b}_{0,0}^{0} \cdots \hat{b}_{m_{t}, m_{x}}^{0} 0 \cdots 0\right]^{\mathrm{T}}
$$

using for instance the LS-PDE method proposed in [12]. In this first step the system is considered as a LTSI system.

- Step 2 Compute a first estimated filter on the basis of the estimates obtained in Step 1

$$
\mathcal{Q}\left(\check{\partial}, \hat{\theta}^{0}\right)=\frac{1}{F\left(\check{\partial}, \hat{\theta}^{0}\right)}
$$

where $F\left(ð, \hat{\theta}^{0}\right)$ is given as (12).

Use the filter in order to generate the estimated derivatives

$$
\begin{gathered}
\left\{u_{l, \mathrm{f}}^{\left(j_{t}, j_{x}\right)}\right\}_{j_{t}=0, j_{x}=0, l=0}^{m_{t}, m_{x}, m_{\beta}}=\mathcal{Q}_{c}\left(ð, \hat{\theta}^{0}\right)\left\{u_{l}^{\left(j_{t}, j_{x}\right)}\right\}_{j_{t}=0, j_{x}=0, l=0}^{m_{t}, m_{x}, m_{\beta}} \\
\left\{y_{\mathrm{f}}^{\left(i_{t}, i_{x}\right)}\right\}_{i_{t}=0, i_{x}=0}^{n_{t}, n_{x}}=\mathcal{Q}_{c}\left(ð, \hat{\theta}^{0}\right)\left\{y^{\left(i_{t}, i_{x}\right)}\right\}_{i_{t}=0, i_{x}=0}^{n_{t}, n_{x}}
\end{gathered}
$$

and the regressor $\varphi_{\mathrm{f}}\left(t_{k}, x_{\ell}\right)$ built up from the filtered noisy output only and the input for this initialization step

$$
\begin{aligned}
\varphi_{\mathrm{f}}\left(t_{k}, x_{\ell}\right)= & {\left[-y_{\mathrm{f}}\left(t_{k}, x_{\ell}\right) \cdots-y_{\mathrm{f}}^{\left(n_{t}, n_{x}-1\right)}\left(t_{k}, x_{\ell}\right)\right.} \\
- & y_{1, \mathrm{f}}^{(0,0)}\left(t_{k}, x_{\ell}\right) \cdots-y_{n_{\alpha}, \mathrm{f}}^{\left(n_{n}, n_{x}-1\right)}\left(t_{k}, x_{\ell}\right) \\
& \left.u_{0, \mathrm{f}}^{(0,0)}\left(t_{k}, x_{\ell}\right) \cdots u_{m_{\beta}, \mathrm{f}}^{\left(m_{t}, m_{x}\right)}\left(t_{k}, x_{\ell}\right)\right]^{\mathrm{T}}
\end{aligned}
$$

- step 3 Compute an estimate $\hat{\theta}^{1}$ :

$$
\begin{aligned}
& \hat{\theta}^{1}=\left[\sum_{k, \ell=1}^{N, L} \varphi_{\mathrm{f}}\left(t_{k}, x_{\ell}\right) \varphi_{\mathrm{f}}^{\mathrm{T}}\left(t_{k}, x_{\ell}\right)\right]^{-1} . \\
& {\left[\sum_{k, \ell=1}^{N, L} \varphi_{\mathrm{f}}\left(t_{k}, x_{\ell}\right) y_{\mathrm{f}}^{\left(n_{t}, n_{x}\right)}\left(t_{k}, x_{\ell}\right)\right] }
\end{aligned}
$$

Set $\tau=1$. [End of initialization part.]

- Step 4 At any iteration $\tau+1$, compute an estimate of the

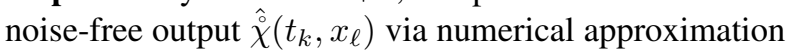
of

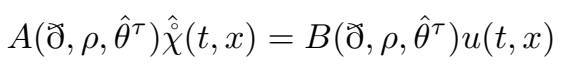

based on the estimates $\hat{\theta}^{\tau}$ at the previous iteration.

- Step 5 Compute the estimated filters

$$
\mathcal{Q}\left(ð, \hat{\theta}^{\tau}\right)=\frac{1}{F\left(\check{\partial}, \hat{\theta}^{\tau}\right)}
$$

and use the filters as well as $\hat{\grave{\chi}}\left(t_{k}, x_{\ell}\right)$ in order to generate the estimates of the derivatives:

$$
\left\{\hat{\mathrm{o}}_{l, \mathrm{f}}^{\left(i_{t}, i_{x}\right)}\right\}=\mathcal{Q}\left(ð, \hat{\theta}^{\tau}\right)\left\{\hat{\mathrm{o}}_{l}^{\left(i_{t}, i_{x}\right)}\right\}
$$

for $i_{t}=0, \cdots, n_{t} ; i_{x}=0, \cdots, n_{x}$ and $l=1, \cdots, n_{\alpha}$

$$
\left\{u_{l, \mathrm{f}}^{\left(j_{t}, j_{x}\right)}\right\}=\mathcal{Q}\left(ð, \hat{\theta}^{\tau}\right)\left\{u_{l}^{\left(j_{t}, j_{x}\right)}\right\}
$$

for $j_{t}=0, \cdots, m_{t} ; j_{x}=0, \cdots, m_{x}$ and $l=$ $1, \cdots, m_{\beta}$

$$
\left\{y_{\mathrm{f}}^{\left(i_{t}, i_{x}\right)}\right\}=\mathcal{Q}\left(\check{\partial}, \hat{\theta}^{\tau}\right)\left\{y^{\left(i_{t}, i_{x}\right)}\right\}
$$

for $i_{t}=0, \cdots, n_{t}$ and $i_{x}=0, \cdots, n_{x}$.

- Step 6 Build the estimated filtered regressor $\hat{\varphi}_{\mathrm{f}}\left(t_{k}, x_{\ell}\right)$ as:

$$
\begin{aligned}
& \hat{\varphi}_{\mathrm{f}}\left(t_{k}, x_{\ell}\right)=\left[-y_{\mathrm{f}}^{(0,0)}\left(t_{k}, x_{\ell}\right) \cdots-y_{\mathrm{f}}^{\left(n_{t}, n_{x}-1\right)}\left(t_{k}, x_{\ell}\right)\right. \\
& -\hat{\stackrel{o}{\chi}}_{1, \mathrm{f}}^{(0,0)}\left(t_{k}, x_{\ell}\right) \cdots-\hat{\mathrm{o}}_{n_{\alpha}, \mathrm{f}}^{\left(n_{t}, n_{x}-1\right)}\left(t_{k}, x_{\ell}\right) \\
& \left.u_{0, \mathrm{f}}^{(0,0)}\left(t_{k}, x_{\ell}\right) \cdots u_{m_{\beta}, \mathrm{f}}^{\left(m_{t}, m_{x}\right)}\left(t_{k}, x_{\ell}\right)\right]^{\mathrm{T}}
\end{aligned}
$$

$\hat{\varphi}_{\mathrm{f}}\left(t_{k}, x_{\ell}\right)$ is composed of the filtered noisy output, the filtered noise-free estimated output and the input.

And build the filtered estimated IV $\hat{\zeta}_{\mathrm{f}}\left(t_{k}, x_{\ell}\right)$ as:

$$
\begin{aligned}
& \hat{\zeta}_{\mathrm{f}}\left(t_{k}, x_{\ell}\right)=\left[-\hat{\grave{\partial}}_{\mathrm{f}}^{(0,0)}\left(t_{k}, x_{\ell}\right) \cdots-\hat{\grave{\partial}}_{\mathrm{f}}^{\left(n_{t}, n_{x}-1\right)}\left(t_{k}, x_{\ell}\right)\right. \\
& -\hat{\grave{\chi}}_{1, \mathrm{f}}^{(0,0)}\left(t_{k}, x_{\ell}\right) \cdots-\hat{\hat{\chi}}_{n_{\alpha}, \mathrm{f}}^{\left(n_{t}, n_{x}-1\right)}\left(t_{k}, x_{\ell}\right) \\
& \left.u_{0, \mathrm{f}}^{(0,0)}\left(t_{k}, x_{\ell}\right) \cdots u_{m_{\beta}, \mathrm{f}}^{\left(m_{t}, m_{x}\right)}\left(t_{k}, x_{\ell}\right)\right]^{\mathrm{T}}
\end{aligned}
$$

$\hat{\zeta}_{\mathrm{f}}\left(t_{k}, x_{\ell}\right)$ vector is composed of the filtered noisefree estimated output and the input and represents the instruments.

- Step 7 Compute the LPV-SRIVC-PDE estimate $\hat{\theta}^{\tau+1}$ at the $(\tau+1)$ th iteration:

$$
\begin{aligned}
& \hat{\theta}^{\tau+1}=\left[\sum_{k, \ell=1}^{N, L} \hat{\zeta}_{\mathrm{f}}\left(t_{k}, x_{\ell}\right) \hat{\varphi}_{\mathrm{f}}^{\mathrm{T}}\left(t_{k}, x_{\ell}\right)\right]^{-1} \cdot \\
& {\left[\sum_{k, \ell=1}^{N, L} \hat{\zeta}_{\mathrm{f}}\left(t_{k}, x_{\ell}\right) y_{\mathrm{f}}^{\left(n_{t}, n_{x}\right)}\left(t_{k}, x_{\ell}\right)\right] }
\end{aligned}
$$

- Step 8 If $\hat{\theta}^{\tau+1}$ has converged or the maximum number of iterations is reached, then stop, else increase $\tau$ by 1 and go to Step 4.

\section{NUMERICAL EXAMPLE}

The performance of the described algorithm is evaluated in the case of the advection-diffusion equation (ADE). This equation is often used in the water resource quality analysis to describe the transport and dispersion of a solute into a river channel [15], [3]. The ADE takes the following form:

$$
\left\{\begin{array}{l}
\frac{\partial \dot{\chi}(t, x)}{\partial t}=a_{02}(\rho(t)) \frac{\partial^{2} \dot{\chi}(t, x)}{\partial x^{2}}-a_{01}(\rho(t)) \frac{\partial \dot{\chi}(t, x)}{\partial x} \\
\dot{\chi}(t=0, x)=0 \\
y\left(t_{k}, x_{\ell}\right)=\stackrel{\chi}{\chi}\left(t_{k}, x_{\ell}\right)+e\left(t_{k}, x_{\ell}\right)
\end{array}\right.
$$

The noise-free signal $\dot{\chi}\left(x_{\ell}, t_{k}\right)$ (Fig. 1) represents the solute propagation in the river and is obtained by discretization of the partial differential equation by a finite difference method (see e.g. [10]).

The temporal sampling is $T_{\mathrm{s}}=10 \mathrm{~s}$ and $N=8640$ temporal points are measured. The spatial sampling is $h=10 \mathrm{~m}$ for $L=61$ space points. These 61 points can represent 61 sensors distributed in the river. $u(t, x)$ describes the source 


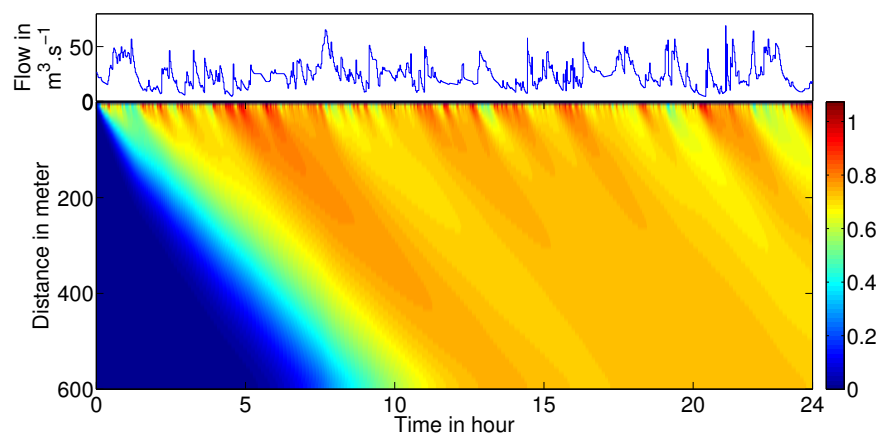

Fig. 1. River flow $\rho(t)$ and noise-free output $\stackrel{\circ}{\chi}(t, x)$

of the pollutant, and is represented by a PRBS signal at the space point $x=20 \mathrm{~m}$. In realistic conditions of simulation the $a_{02}(\rho(t))$ diffusion and the $a_{01}(\rho(t))$ advection coefficients are dependent of the river flow $Q(t)$ (Fig. 1). The scheduling variable $\rho$ is then taken as $\rho(t)=Q(t)$ and the coefficients $a_{02}(\rho(t)), a_{01}(\rho(t))$ and $b_{00}(\rho(t))$ are given by

$$
\left\{\begin{array}{l}
\stackrel{\circ}{a}_{02}(\rho(t))=532+7.3 \rho(t) \\
\stackrel{\circ}{a}_{01}(\rho(t))=52+0.72 \rho(t) \\
\stackrel{\circ}{b}_{00}(\rho(t))=1
\end{array}\right.
$$

For model identification purposes, the output signal is supposed to be corrupted by a two dimensional, zero-mean and normally distributed, discrete-time noise signal, with a Signal-to-Noise Ratio (SNR) defined as

$$
S N R=10 \log _{10}\left(\frac{P_{\dot{\chi}}}{P_{e}}\right)
$$

where $P_{e}$ and $P_{\chi}$ are the average power of the noise and deterministic output.

To provide representative results, Monte Carlo simulation analysis for 50 runs are used to illustrate the performance of the approach. Table I and II display the estimated parameters when the additive noise is white and colored respectively. In the colored case, the additive colored noise $v\left(t_{k}, x_{\ell}\right)$ is done by:

$$
v\left(t_{k}, x_{\ell}\right)=\frac{1}{1-1.2 q_{t}^{-1}+0.4 q_{t}^{-2}} e\left(t_{k}, x_{\ell}\right) .
$$

The parameters are estimated by the proposed approach denoted as SRIVC and the LPV-SILS-PDE developed in [13] and denoted as SILS (for simplified iterative least squares). From both Tables, it can be noted that the estimates of the proposed SRIVC method are unbiased with relatively small standard deviations. As expected, the estimates delivered by the SILS method are unbiased only when the output is corrupted by a white noise.

\section{CONCLUSION}

A refined instrumental variable algorithm has been presented to estimate linear parameter-varying partial derivative equations. The implied continuous filtering operations are rewritten under a discrete transfer function form. Based on a numerical example, the proposed method delivered unbiased estimated parameters whatever the noise structure while the
TABLE I

MC SIMULATION RESUlTS, WHITE NOISE, $S N R=25 d B$

\begin{tabular}{ccccccc}
\hline & & $\hat{a}_{02}^{0}$ & $\hat{a}_{02}^{1}$ & $\hat{a}_{01}^{0}$ & $\hat{a}_{01}^{1}$ & $\hat{b}_{00}^{0}$ \\
\hline method & True value & 532 & 7.3 & 52 & 0.72 & 1 \\
\hline SILS & mean & 534.2 & 7.263 & 51.85 & 0.7263 & 1.001 \\
iter=7 & std & 1.2 & 0.042 & 0.08 & 0.0035 & 0.001 \\
\hline SRIVC & mean & 533.4 & 7.321 & 51.98 & 0.7207 & 1.001 \\
iter=8 & std & 1.2 & 0.0419 & 0.08 & 0.0035 & 0.001 \\
\hline
\end{tabular}

TABLE II

MC SIMULATION RESULTS, COLORED NOISE, $S N R=25 d B$

\begin{tabular}{ccccccc}
\hline & & $\hat{a}_{02}^{0}$ & $\hat{a}_{02}^{1}$ & $\hat{a}_{01}^{0}$ & $\hat{a}_{01}^{1}$ & $\hat{b}_{00}^{0}$ \\
\hline method & True value & 532 & 7.3 & 52 & 0.72 & 1 \\
\hline SILS & mean & 392.6 & 9.7476 & 58.68 & 0.4313 & 0.937 \\
iter= 7 & std & 4.9 & 0.1729 & 0.34 & 0.0148 & 0.003 \\
\hline SRIVC & mean & 530.8 & 7.2991 & 52.02 & 0.7184 & 0.999 \\
iter=8 & std & 5.3 & 0.1811 & 0.35 & 0.0154 & 0.004 \\
\hline
\end{tabular}

iterative least squares method gave biased estimates when the additive noise was colored. Future research investigates the case where few sensors are available for the reconstruction of the noise-free output.

\section{REFERENCES}

[1] M. Ali, A. Ali, H. Abbas, and H. Werner, "Identification of BoxJenkins models for parameter-varying spatially interconnected systems", American Control Conference, San Francisco (USA), pp: 145$150,2011$.

[2] G. Belforte, F. Dabbene, and P. Gay, "LPV approximation of distributed parameter systems in environmental modelling", Environmental Modelling \& Software, vol. 20, pp: 1063-1070, 2005.

[3] K. E. Bencala, and R. A. Walters, "Simulation of solute transport in a mountain pool-and-rifle stream: a transient storage model", Water Resources Research, vol. 19(3), pp: 718-724, 1983.

[4] C. Chochol, S. Chesne, and D. Remond, "An original differentiation tool for identification on continuous structures", Journal of Sound and Vibration, 2013.

[5] R. F. Curtain, and K. Morris, "Transfer function of distributed parameter systems : A tutorial", Automatica, vol. 45, pp: 11011116, 2009.

[6] C. S. Kubrusly, "Distributed parameter system identification A Survey", International Journal of Control vol. 26(4), 509-535, 1977.

[7] V. Laurain, R. Tóth, M. Gilson, and H. Garnier, " Direct identification of continuous-time linear parameter-varying input/output models", IET Control Theory \& Applications, vol. 5(7), pp: 878-888, 2011.

[8] P. Lohnberg and G. H. J. Wisselink, "Iterative Least-Squares Parameter Estimation for ARMA Pulse Response and Output Disturbance", IEEE Transactions on Automatique Control, 27(6), pp: 1252-1255, 1982.

[9] R. Malti, M. Aoun, J. Sabatier, and A. Oustaloup, "Tutorial on system identification using fractional differentiation models", In 14th IFAC Symposium on System Identification, pp: 606-611, Newcastle (Australia), 2006.

[10] K. W. Morton and D. F. Mayers, Numerical Solution of Partial Differential Equations, Cambridge University Press, 2005.

[11] M. S. Sadabadi, M. Shafiee, and M. Karrari, "System identification of two dimensional continuous-time systems using wavelets as modulating functions", ISA Transactions, vol. 47, pp: 256-266, 2008.

[12] J. Schorsch, H. Garnier, M. Gilson, and P. C. Young, "Instrumental variable methods for identifying partial differential equation models", International Journal of Control, in press, 2013.

[13] J. Schorsch, M. Gilson, V. Laurain, and H. Garnier, "Identification of LPV partial differential equation models", 52nd IEEE Conference on Decision and Control, Florence (Italy), 2013.

[14] T. Söderström and P. Stoica, Instrumental Variable Methods for System Identification, New York: Springer Verlag (1983).

[15] W. Tych and P.C. Young, "A matlab software framework for dynamic model emulation", Environmental Modelling and Software, vol. 34, pp: 19-29, 2012.

[16] P.C. Young, Recursive Estimation and Time-series Analysis: An Introduction for the Student and Practitioner, second ed., Berlin: SpringerVerlag, 2011. 